International Journal of Pure and Applied Mathematics

Volume 102 No. 2 2015, 357-382

ISSN: 1311-8080 (printed version); ISSN: 1314-3395 (on-line version)

url: http://www.ijpam.eu

doi: http://dx.doi.org/10.12732/ijpam.v102i2.13

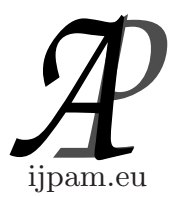

\title{
THE MODIFIED JUNGCK MANN AND MODIFIED \\ JUNGCK ISHIKAWA ITERATION SCHEMES FOR ZAMFIRESCU OPERATORS
}

\author{
Arif Rafiq ${ }^{1}$, Muhammad Tanveer ${ }^{2}$, Shin Min Kang ${ }^{3} \S$ \\ ${ }^{1,2}$ Department of Mathematics \\ Lahore Leads University \\ Lahore, 54810, PAKISTAN \\ ${ }^{3}$ Department of Mathematics and RINS \\ Gyeongsang National University \\ Jinju, 660-701, KOREA
}

\begin{abstract}
The purpose of this paper is to establish some strong convergence results of the modified Jungck Mann and modified Jungck Ishikawa iteration schemes to a common fixed point for Zamfirescu operators.
\end{abstract}

AMS Subject Classification: 47J25, 54H25, 65A05

Key Words: modified Jungck Mann iteration scheme, modified Jungck Ishikawa iteration scheme, Zamfirescu operator

\section{Introduction}

Let $(E,\|\cdot\|)$ be a Banach space and $T: E \rightarrow E$ be a self mapping of $E$.

There are many iteration schemes for which the fixed points of operators have been approximated in last few decades by various authors.

Received: April 25, 2015

(c) 2015 Academic Publications, Ltd.

$\S_{\text {Correspondence author }}$ url: www.acadpubl.eu 
Now, we shall state some of these iteration schemes as follows:

Let $(X, d)$ be a metric space and $T: X \rightarrow X$ be a self mapping. Choose $x_{0} \in X$ and define $x_{1}=T x_{0}, x_{2}=T x_{1}, \cdots$, and obtain a relation

$$
x_{n+1}=T x_{n}, \quad n=0,1,2, \ldots,
$$

where $x_{n}$ is the $n$-th Picard iterate of $x_{0} \in X$.

Let $x_{0}$ be an initial point in a given set and $\left\{\alpha_{n}\right\}$ with $0 \leq \alpha_{n}<1$, be a sequence of real numbers. Then Mann iteration scheme [7] is defined by

$$
x_{n+1}=\left(1-\alpha_{n}\right) x_{n}-\alpha_{n} T x_{n}, \quad n=0,1,2, \ldots
$$

Let $x_{0}$ be an initial point in a given set and $\left\{\alpha_{n}\right\}$ and $\left\{\beta_{n}\right\}$ be the sequences of real numbers with $0 \leq \alpha_{n} \leq \beta_{n}<1$. Then

$$
\begin{aligned}
x_{n+1} & =\left(1-\alpha_{n}\right) x_{n}+\alpha_{n} T y_{n} \\
y_{n} & =\left(1-\beta_{n}\right) x_{n}+\beta_{n} T x_{n}, \quad n=0,1,2, \ldots
\end{aligned}
$$

is called Ishikawa iteration scheme [3].

Berinde [1] proved the strong convergence results in an arbitrary Banach space for the Ishikawa iteration scheme by using the following contractive definitions:

Let $(E,\|\cdot\|)$ be a Banach space and $T: E \rightarrow E$ a self mapping of $E$, there exist $a, b$ and $c$ satisfying $0 \leq a<1,0 \leq b<\frac{1}{2}$ and $0 \leq c<\frac{1}{2}$ respectively such that for each $x, y \in E$, at least one of the following is true:

$$
\begin{gathered}
d(T x, T y) \leq a d(x, y), \\
d(T x, T y) \leq b[d(x, T x)+d(y, T y)], \\
d(T x, T y) \leq c[d(x, T y)+d(y, T x)] .
\end{gathered}
$$

(1.3), (1.4) and (1.5) are called Zamfirescu contraction conditions and the mapping satisyfing these conditions is called Zamfirescu operator [11]. The mapping satisfying (1.3) is called Kannan operator [6] and the mapping satisfying (1.4) is called Chattererjea operator [2]. 


\section{Preliminaries}

We shall use the following fundamental results and iteration schemes in establishing our main results:

Let $(X,\|\cdot\|)$ be a normed linear space and $S, T: S \longrightarrow E$ are two non-self mappings such that $T(Y) \subseteq S(Y)$. Then the sequence $\left\{S x_{n}\right\}_{n=0}^{\infty}$ defined by

$$
S x_{n+1}=T x_{n}, \quad n=0,1,2 \ldots
$$

is called Jungck iteration scheme [4].

The following iteration scheme is introduced by Singh et al. [10] to establish some stability results:

Let $(X,\|\cdot\|)$ be a normed linear space and $S, T: S \longrightarrow E$ are two non-self mappings such that $T(Y) \subseteq S(Y)$. Then the sequence $\left\{S x_{n}\right\}_{n=0}^{\infty}$ defined by

$$
S x_{n+1}=\left(1-\alpha_{n}\right) S x_{n}+\alpha_{n} T x_{n}, \quad n=0,1,2, \ldots,
$$

where $\left\{\alpha_{n}\right\}$ is the sequence in $[0,1)$, and is called Jungck Mann iteration scheme.

Let $(X,\|\cdot\|)$ be a normed linear space and $S, T: S \longrightarrow E$ are two non-self mappings such that $T(Y) \subseteq S(Y)$. Then the sequence $\left\{S x_{n}\right\}_{n=0}^{\infty}$ defined by

$$
\begin{aligned}
S x_{n+1} & =\left(1-\alpha_{n}\right) S x_{n}+\alpha_{n} T z_{n} \\
S z_{n} & =\left(1-\beta_{n}\right) S x_{n}+\beta_{n} T x_{n}, \quad n=0,1,2, \ldots,
\end{aligned}
$$

where $\left\{\alpha_{n}\right\}$ and $\left\{\beta_{n}\right\}$ are the sequences in $[0,1)$. This is called Jungck Ishikawa iteration scheme [8]. If $X=Y$ or $S$ is the identity mapping, then Jungck Picard, Jungck Mann and Jungck Ishikawa iteration schemes becomes Picard, Mann and Ishikawa iteration schemes.

Olatinwo and Imoru [9] used the the following definitions and contractive conditions:

Definition 2.1. Let $(X, d)$ be a metric space and $T: X \rightarrow X$ be a self mapping. Then $T$ has a fixed point if there is an $x \in X$ such that $T x=x$. The point $x$ is called a fixed point of $T$.

Definition 2.2. Let $(X, d)$ be a metric space and $T, S: X \rightarrow X$. Then $x$ is called a coincidence (common fixed) point of $T$ and $S$, respectively, if there exists $x \in X$ such that

$$
x=T x=S x .
$$

Definition 2.3. If an operator $T$ satisfied above three conditions (1.3)(1.5), then it is called Zamfirescu operator. For two non-self mappings $S, T$ : 
$Y \rightarrow E$ with $T(Y) \subseteq S(Y)$, there exist real numbers $\alpha, \beta$ and $\gamma$ satisfying $0 \leq \alpha<1,0 \leq \beta<1 / 2$ and $0 \leq \gamma<\frac{1}{2}$, respectively such that for each $x, y \in Y$, at least one of the following is true.

$$
\begin{gathered}
d(T x, T y) \leq \alpha d(S x, S y), \\
d(T x, T y) \leq \beta[d(S x, T x)+d(S y, T y)], \\
d(T x, T y) \leq \gamma[d(S x, T y)+d(S y, T x)] .
\end{gathered}
$$

The above three conditions will be called the generalized Zamfirescu contraction for the pair $(S, T)$. Condition (2.4) is called generlized Kannan condition for the pair of $(S, T)$ and condition (2.5) is called of Chatterjea condition for the pair of $(S, T)$.

In this paper, we purposed the modified Jungck Mann and modified Jungck Ishikawa iteration schemes for Zamfirescu operators for solving nonlinear functions by following the approach of [5]. We also provided the applications of the two iteration schemes and compared them with the Jungck iteration scheme, Jungck Mann iteration scheme and the Jungck Ishikawa iteration scheme by using some significant nonlinear functions.

\section{Main Results}

Consider the nonlinear equation

$$
f(x)=0, \quad x \in \mathbb{R} .
$$

Let $S, T: Y \rightarrow X, T(Y) \subset S(Y), S$ is onto and $T$ is differentiable. Suppose that $\xi$ is simple zero of $f(x)$ and $x_{0}$ is an initial guess nearer to $\xi$. The equation (3.1) can be written as

$$
S x=T x .
$$

Following the approch of [5], if $T^{\prime} x \neq-1$, we can modify (3.2) by multiplying $\theta \neq-1$ on both sides as follows

$$
\theta S x=\theta T x,
$$

which implies that

$$
S x=\frac{\theta T x+S x}{\theta+1},
$$


where $\theta$ is an orbitrary number. In order (3.3) to be efficient, we can choose

$$
\theta=-T^{\prime} x .
$$

For a given $x_{0}$, we can find the approximate solution of $x_{n+1}$ by using the iteration scheme named as modified Jungck iteration scheme

$$
S x_{n+1}=\frac{\theta T x_{n}+S x_{n}}{\theta+1}, \quad \theta \neq-1,
$$

or

$$
T x_{n+1}=\frac{\theta T x_{n}+S x_{n}}{\theta+1}, \quad \theta \neq-1 .
$$

Using (3.6) in Jungck Mann and Jungck Ishikawa iteration schemes, we develope modified Jungck Mann iteration scheme and modified Jungck Ishikawa iteration scheme. modified Jungck Mann iteration scheme and modified Jungck Ishikawa iteration scheme are as follows:

$$
S x_{n+1}=\left(1-\alpha_{n}\right) S x_{n}+\alpha_{n} T_{\theta} x_{n},
$$

and

$$
\begin{aligned}
S x_{n+1} & =\left(1-\alpha_{n}\right) S x_{n}+\alpha_{n} T_{\theta} z_{n}, \\
S z_{n} & =\left(1-\beta_{n}\right) S x_{n}+\beta_{n} T x_{n},
\end{aligned}
$$

where $T_{\theta} x_{n}=\frac{\theta T x_{n}+S x_{n}}{1+\theta}$ and $T_{\theta} z_{n}=\frac{\theta T z_{n}+S z_{n}}{1+\theta}, \theta \neq-1,\left\{\alpha_{n}\right\}_{n=0}^{\infty}$ and $\left\{\beta_{n}\right\}_{n=0}^{\infty}$ are the sequences in $[0,1)$.

Now we shall establish the following theorems as our main results:

Theorem 3.1. Let $(E,\|\cdot\|)$ be any Banach space and $Y$ be an orbitrary set. Suppose that $S, T: S \longrightarrow E$ are two non-self mappings such that $T(Y) \subseteq S(Y)$, where $S(Y)$ is a complete subspace of $E, S$ is onto and $T$ is differentiable. let $l$ be the coincidence point of $S$ and $T(i . e, S l=T l=w)$. Suppose also that $S$ and $T$ satisfy the contractive conditions (2.1)-(2.3). For $x_{0} \in Y$, let $\left\{S x_{n}\right\}_{n=0}^{\infty}$ be modified Jungck Mann iteration scheme defined by (3.7), where $\left\{\alpha_{n}\right\}_{n=0}^{\infty}$ is the sequence in $[0,1)$ such that $\sum_{k=0}^{\infty} \alpha_{k}=\infty$. Then $\left\{S x_{n}\right\}_{n=0}^{\infty}$ strongly converges to $w$.

Proof. We shall first establish that the conditions (2.3)-(2.5) implies

$$
\begin{aligned}
& \left\|T_{\theta} x-T_{\theta} y\right\| \\
& \leq \beta\left[\left\|S x-T_{\theta} x\right\|+\left\|S y-T_{\theta} y\right\|\right] \\
& \leq \beta\left[\left\|S x-T_{\theta} x\right\|+\|S y-S x\|+\left\|S x-T_{\theta} x\right\|+\left\|T_{\theta} x-T_{\theta} y\right\|\right],
\end{aligned}
$$


which implies that

$$
\left\|T_{\theta} x-T_{\theta} y\right\| \leq\left(\frac{2 \beta}{1-\beta}\right)\left\|S x-T_{\theta} x\right\|+\left(\frac{\beta}{1-\beta}\right)\|S y-S x\| .
$$

Similary

$$
\left\|T_{\theta} x-T_{\theta} y\right\| \leq\left(\frac{2 \gamma}{1-\gamma}\right)\left\|S x-T_{\theta} x\right\|+\left(\frac{\gamma}{1-\gamma}\right)\|S y-S x\| .
$$

From (3.9) and (3.10)

$$
\left\|T_{\theta} x-T_{\theta} y\right\| \leq 2 \delta\left\|S x-T_{\theta} x\right\|+\delta\|S y-S x\|,
$$

where $\delta=\max \left\{\alpha, \frac{\beta}{1-\beta}, \frac{\gamma}{1-\gamma}\right\}$ for $0 \leq \delta<1$. Let $C(S, T)$ be the set of coincedence points $S$ and $T$. We shall now use the (3.11) to establish that $S$ and $T$ have a unique coincedence point $l$ (i.e, $S l=T l=w$ ). Injectivity of $S$ is sufficient. Suppose that there exist $l_{1}, l_{2} \in C(S, T)$ such that $S\left(l_{1}\right)=T\left(l_{1}\right)=w_{1}$ and $S\left(l_{2}\right)=T\left(l_{2}\right)=w_{2}$. If $w_{1}=w_{2}$, then $S\left(l_{1}\right)=S\left(l_{2}\right)$ and since $\mathrm{S}$ is onto, it follows that $l_{1}=l_{2}$.

If $w_{1} \neq w_{2}$ by using (3.10) for $S$ and $T$, then

$$
\begin{aligned}
0 & <\left\|w_{1}-w_{2}\right\| \\
& =\left\|T_{\theta}\left(l_{1}\right)-T_{\theta}\left(l_{2}\right)\right\| \\
& \leq 2 \delta\left\|S\left(l_{1}\right)-T_{\theta}\left(l_{1}\right)\right\|+\delta\left\|S\left(l_{2}\right)-S\left(l_{1}\right)\right\| \\
& =\delta\left\|w_{2}-w_{1}\right\|,
\end{aligned}
$$

which implies $(1-\delta)\left\|w_{2}-w_{1}\right\| \leq 0$, it follows that $(1-\delta)>0$ for $\delta \in[0,1)$, but $\left\|w_{2}-w_{1}\right\| \leq 0$, which contradicts that norm is nonnegative. Therefore, we have $\left\|w_{2}-w_{1}\right\|=0$, i.e, $w_{1}=w_{2}=w$. Since $w_{1}=w_{2}$, then we have $w_{1}=S\left(l_{1}\right)=T\left(l_{1}\right)=S\left(l_{2}\right)=T\left(l_{2}\right)=w_{2}$, implies that $S\left(l_{1}\right)=S\left(l_{2}\right)$, leading to $l_{1}=l_{2}=l$ (since $S$ is onto). Hence $S$ and $T$ have a unique coincedence point $l \in C(S, T)$. Using $\delta=\max \left\{\alpha, \frac{\beta}{1-\beta}, \frac{\gamma}{1-\gamma}\right\}, 0 \leq \delta<1$ and $\theta \neq-1$. Put $y=x_{n}$ and $x=w$ in (3.11), we have

$$
\begin{aligned}
\left\|T_{\theta} x_{n}-w\right\| & \leq 2 \delta\left\|S w-T_{\theta} w\right\|+\delta\left\|S x_{n}-w\right\| \\
& \leq 2 \delta\left\|S w-\frac{\theta T w+S w}{1+\theta}\right\|+\delta\left\|S x_{n}-w\right\| \\
& \leq 2 \delta\left\|\frac{S w+\theta S w-\theta T w-S w}{1+\theta}\right\|+\delta\left\|S x_{n}-w\right\|,
\end{aligned}
$$


which implies

$$
\left\|T_{\theta} x_{n}-w\right\| \leq \delta\left\|S x_{n}-w\right\| .
$$

We now prove that $\left\{S x_{n}\right\}_{n=0}^{\infty}$ strongly converges to $w$, where $S l=T l=w$, using again (3.11). Therefore, we have

$$
\begin{aligned}
& \left\|S x_{n+1}-w\right\|=\left\|\left(1-\alpha_{n}\right) S x_{n}+\alpha_{n} T_{\theta} x_{n}-w\right\| \\
& \leq\left(1-\alpha_{n}\right)\left\|S x_{n}-w\right\|+\alpha_{n}\left\|T_{\theta} x_{n}-w\right\| \\
& \leq\left(1-\alpha_{n}\right)\left\|S x_{n}-w\right\|+\delta \alpha_{n}\left\|S x_{n}-w\right\| \\
& \leq\left[1-(1-\delta) \alpha_{n}\right]\left\|S x_{n}-w\right\| \\
& \leq \prod_{k=0}^{n}\left[1-(1-\delta) \alpha_{k}\right]\left\|S x_{0}-w\right\| \\
& \leq e^{-\left[(1-\delta) \sum_{k=0}^{n} \alpha_{k}\right]}\left\|S x_{0}-w\right\| \\
& \rightarrow 0 \text { as } n \rightarrow \infty \text {, }
\end{aligned}
$$

since $\sum_{k=0}^{n} \alpha_{k}=\infty, \delta \in[0,1)$ and $\theta \neq-1$. Therefore, from (3.13) we get $\left\|S x_{n}-w\right\| \longrightarrow 0$ as $n \rightarrow \infty$, that is, $\left\{S x_{n}\right\}_{n=0}^{\infty}$ strongly converges to $w$. This compleets the proof.

Theorem 3.2. Let $(E,\|\cdot\|)$ be any Banach space and $Y$ be an orbitrary set. Suppose that $S, T: S \longrightarrow E$ are two non-self mappings such that $T(Y) \subseteq S(Y)$, where $S(Y)$ is a complete subspace of $E, S$ is onto and $T$ is differentiable. let $l$ be the coincidence point of $S$ and $T$ (i.e., $S l=T l=w$ ). Suppose also that $S$ and $T$ satisfy the contractive conditions (2.3)-(2.5). For $x_{0} \in Y$, let $\left\{S x_{n}\right\}_{n=0}^{\infty}$ be modified Jungck Ishikawa iteration scheme defined by (3.8), where $\left\{\alpha_{n}\right\}_{n=0}^{\infty}$ and $\left\{\beta_{n}\right\}_{n=0}^{\infty}$ are the sequences in $[0,1)$ such that $\sum_{k=0}^{\infty} \alpha_{k}=\infty$. Then $\left\{S x_{n}\right\}_{n=0}^{\infty}$ strongly converges to $w$.

Proof. Let us suppose that $S x_{n+1}=\left(1-\alpha_{n}\right) S x_{n}+\alpha_{n} T_{\theta} z_{n}$, where $S z_{n}=$ $\left(1-\beta_{n}\right) S x_{n}+\beta_{n} T x_{n}, T_{\theta} x_{n}=\frac{\theta T x_{n}+S x_{n}}{1+\theta}$ and $T_{\theta} z_{n}=\frac{\theta T z_{n}+S z_{n}}{1+\theta}, \theta \neq-1$. Then using condition (3.11), we have

$$
\begin{aligned}
\left\|S x_{n+1}-w\right\| & =\left\|\left(1-\alpha_{n}\right) S x_{n}+\alpha_{n} T_{\theta} z_{n}-w\right\| \\
& \leq\left(1-\alpha_{n}\right)\left\|S x_{n}-w\right\|+\alpha_{n}\left\|T_{\theta} z_{n}-w\right\| \\
& \leq\left(1-\alpha_{n}\right)\left\|S x_{n}-w\right\|+\delta \alpha_{n}\left\|S z_{n}-w\right\| .
\end{aligned}
$$

Also, we have

$$
\begin{aligned}
\left\|S z_{n}-w\right\| & \leq\left(1-\beta_{n}\right)\left\|S x_{n}-w\right\|+\beta_{n}\left\|T x_{n}-w\right\| \\
& \leq\left(1-\beta_{n}\right)\left\|S x_{n}-w\right\|+\delta \beta_{n}\left\|S x_{n}-w\right\| \\
& =\left[\left(1-(1-\delta) \beta_{n}\right]\left\|S x_{n}-w\right\| .\right.
\end{aligned}
$$


Using (3.15) in (3.14) yields

$$
\begin{aligned}
& \left\|S x_{n+1}-w\right\| \\
& \left.\leq\left(1-\alpha_{n}\right) \| S x_{n}-w\right)\left\|+\delta \alpha_{n}\left[1-\beta_{n}(1-\delta)\right]\right\| S x_{n}-w \| \\
& \leq\left[1-(1-\delta) \alpha_{n}-(1-\delta) \delta \alpha_{n} \beta_{n}\right]\left\|S x_{n}-w\right\| \\
& \left.\leq\left[1-(1-\delta) \alpha_{n}\right]\left\|S x_{n}-w\right\| \quad \text { (because } 1+\delta \beta_{n} \geq 1\right) \\
& \leq \prod_{k=0}^{n}\left[1-(1-\delta) \alpha_{k}\right]\left\|S x_{0}-w\right\| \\
& \leq e^{-\left[(1-\delta) \sum_{k=0}^{n} \alpha_{k}\right]\left\|S x_{0}-w\right\|} \\
& \rightarrow 0 \quad \text { as } n \rightarrow 0,
\end{aligned}
$$

since $\sum_{k=0}^{n} \alpha_{k}=\infty, \alpha \in[0,1)$ and $\theta \neq-1$. Hence, we obtain from (3.16) that $\left\|S x_{n}-w\right\| \rightarrow 0$ as $n \rightarrow \infty$, which implies that $\left\{S x_{n}\right\}_{n=0}^{\infty}$ converges strongly to $w$. This compleets the proof.

\section{Applications}

In this section we present some applications of nonlinear functions that shows the efficiency of modified Jungck Mann and modified Jungck Ishikawa Iteration schemes by comparing them with Jungck, Jungck Mann, Jungck Ishikawa iteration schemes.

Example 4.1. Consider the nonlinear equation $\ln (x)-\cos (x)=0$. Let us take $T x=\ln (x)$ and $S x=\cos (x)$. The true graphical solution of given equation is $1.3029640012(10 D)$. If we choose initial guess $x_{0}=1.5$, then the comparision tables correct upto ten decimal places of Jungck, Jungck Mann, Jungck Ishikawa, modified Jungck Mann and modified Jungck Ishikawa iteration schemes are shown below. From tables, we can see that Jungck iteration scheme evaluate the solution of given equation in 101 iterations, Jungck Mann iteration scheme for $\beta=0.9083$ is diverged, modified Jungck Mann iteration scheme for $\beta=0.9083$ evaluate the solution in 5 iterations, Jungck Ishikawa iteration scheme for $\alpha=0.96636945$ and $\beta=0.3$ evaluate the solution in 23 iterations and modified Jungck Ishikawa iteration scheme for $\alpha=0.96636945$ and $\beta=0.3$ evaluate the solution in 3 iterations of the given equation (see 
Tables 1-5).

Table 1: Jungck iteration scheme

\begin{tabular}{c|c|c|c|c}
\hline$n$ & $T x_{\mathrm{n}}$ & $S x_{\mathrm{n}+1}$ & $x_{\mathrm{n}+1}$ & $\left|e_{\mathrm{n}}\right|$ \\
\hline 0 & 0.4054651081 & 0.4054651081 & 1.1533087441 & 0.3466912559 \\
1 & 0.1426349800 & 0.1426349800 & 1.4276732194 & 0.2743644753 \\
2 & 0.3560459998 & 0.3560459998 & 1.2067631452 & 0.2209100742 \\
3 & 0.1879416885 & 0.1879416885 & 1.3817302578 & 0.1749671126 \\
4 & 0.3233365238 & 0.3233365238 & 1.2415430316 & 0.1401872262 \\
5 & 0.2163549863 & 0.2163549863 & 1.3527168518 & 0.1111738203 \\
6 & 0.3021150529 & 0.3021150529 & 1.2638857195 & 0.0888311323 \\
7 & 0.2341908799 & 0.2341908799 & 1.3344101071 & 0.0705243876 \\
8 & 0.2884893269 & 0.2884893269 & 1.2781476192 & 0.0562624879 \\
9 & 0.2454118573 & 0.2454118573 & 1.3228518075 & 0.0447041883 \\
10 & 0.2797898664 & 0.2797898664 & 1.2872210998 & 0.0356307076 \\
$\vdots$ & $\vdots$ & $\vdots$ & $\vdots$ & $\vdots$ \\
99 & 0.2646416701 & 0.2646416701 & 1.3029640012 & 0.0000000001 \\
100 & 0.2646416702 & 0.2646416702 & 1.3029640012 & 0.0000000000 \\
\hline
\end{tabular}

Table 2: Jungck Mann iteration scheme $(\beta=0.9083)$

\begin{tabular}{c|c|c|c|c}
\hline$n$ & $T x_{\mathrm{n}}$ & $S x_{\mathrm{n}+1}$ & $x_{\mathrm{n}+1}$ & $\left|e_{\mathrm{n}}\right|$ \\
\hline 0 & 0.0707372017 & 0.1014317507 & 1.1067543806 & 0.3932456194 \\
1 & 0.4475662666 & 0.4158257315 & 1.5156217208 & 0.4088673402 \\
2 & 0.0551466162 & 0.0882208911 & 1.0922293591 & 0.4233923617 \\
$\vdots$ & $\vdots$ & $\vdots$ & $\vdots$ & $\vdots$ \\
\hline
\end{tabular}

Table 3: Modified Jungck Mann iteration scheme $(\beta=0.9083)$

\begin{tabular}{c|c|c|c|c}
\hline$n$ & $T x_{\mathrm{n}}$ & $S x_{\mathrm{n}+1}$ & $x_{\mathrm{n}+1}$ & $\left|e_{\mathrm{n}}\right|$ \\
\hline 0 & 0.0707372017 & 0.2536390701 & 1.2887065876 & 0.2112934124 \\
1 & 0.2783633989 & 0.2646412508 & 1.3029634548 & 0.0142568672 \\
2 & 0.2646421971 & 0.2646416728 & 1.3029640046 & 0.0000005498 \\
3 & 0.2646416669 & 0.2646416701 & 1.3029640012 & 0.0000000034 \\
4 & 0.2646416702 & 0.2646416702 & 1.3029640012 & 0.0000000000 \\
\hline
\end{tabular}


Table 4: Jungck Ishikawa iteration scheme $(\alpha=0.96636945$ and $\beta=0.3)$

\begin{tabular}{c|c|c|c|c}
\hline$n$ & $T x_{\mathrm{n}}$ & $S x_{\mathrm{n}+1}$ & $x_{\mathrm{n}+1}$ & $\left|e_{\mathrm{n}}\right|$ \\
\hline 0 & 0.0707372017 & 0.2189661378 & 1.2447891247 & 0.2552108753 \\
1 & 0.3202630995 & 0.2814748656 & 1.3250826907 & 0.0802935660 \\
2 & 0.2432485880 & 0.2586692006 & 1.2952052808 & 0.0298774099 \\
$\vdots$ & $\vdots$ & $\vdots$ & $\vdots$ & $\vdots$ \\
22 & 0.2646416702 & 0.2646416702 & 1.3029640012 & 0.0000000000 \\
\hline
\end{tabular}

Table 5: Modified Jungck Ishikawa iteration scheme $(\alpha=0.96636945$ and $\beta=0.3)$

\begin{tabular}{c|c|c|c|c}
\hline$n$ & $T x_{\mathrm{n}}$ & $S x_{\mathrm{n}+1}$ & $x_{\mathrm{n}+1}$ & $\left|e_{\mathrm{n}}\right|$ \\
\hline 0 & 0.0707372017 & 0.2642115591 & 1.3024037025 & 0.1975962975 \\
1 & 0.2651819509 & 0.2646416701 & 1.3029640012 & 0.0005602987 \\
2 & 0.2646416701 & 0.2646416701 & 1.3029640012 & 0.0000000000 \\
\hline
\end{tabular}

Example 4.2. Consider the nonlinear equation $\ln (x)+\tan (x)=0$. Let us take $T x=-\ln (x)$ and $S x=\tan (x)$. The true graphical solution of given equation is $0.5452257174(10 D)$. If we choose initial guess $x_{0}=0.5$, then the comparision tables correct upto ten decimal places of Jungck, Jungck Mann, Jungck Ishikawa, modified Jungck Mann and modified Jungck Ishikawa iteration schemes are shown below. From tables, we can see that Jungck iteration scheme evaluate the solution of given equation in 74 iterations, Jungck Mann iteration scheme for $\beta=0.66$ evaluate the solution in 37 iterations, modified Jungck Mann iterative scheme for $\beta=0.66$ evaluate the solution in 5 iterations, Jungck Ishikawa iteration scheme for $\beta=0.2879415656$ and $\alpha=0.86$ evaluate the solution in 15 iterations and modified Jungck Ishikawa iteration scheme for $\beta=0.2879415656$ and $\alpha=0.86$ evaluate the solution in 2 iteration of the given equation (see Tables 6-10).

Table 6: Jungck iteration scheme

\begin{tabular}{c|c|c|c|c}
\hline$n$ & $T x_{\mathrm{n}}$ & $S x_{\mathrm{n}+1}$ & $x_{\mathrm{n}+1}$ & $\left|e_{\mathrm{n}}\right|$ \\
\hline 0 & 0.5463024898 & 0.5463024898 & 0.5790870369 & 0.0790870369 \\
1 & 0.6538643799 & 0.6538643799 & 0.5200322865 & 0.0590547504 \\
2 & 0.5726047020 & 0.5726047020 & 0.5640543302 & 0.0440220437 \\
3 & 0.6326119075 & 0.6326119075 & 0.5312025356 & 0.0328517946 \\
$\vdots$ & $\vdots$ & $\vdots$ & $\vdots$ & $\vdots$ \\
72 & 0.6065554097 & 0.6065554097 & 0.5452257174 & 0.0000000001 \\
73 & 0.6065554098 & 0.6065554098 & 0.5452257174 & 0.0000000000 \\
\hline
\end{tabular}


Table 7: Jungck Mann iteration scheme $(\beta=0.66)$

\begin{tabular}{c|c|c|c|c}
\hline$n$ & $T x_{\mathrm{n}}$ & $S x_{\mathrm{n}+1}$ & $x_{\mathrm{n}+1}$ & $\left|e_{\mathrm{n}}\right|$ \\
\hline 0 & 0.6931471806 & 0.6432199857 & 0.5715941783 & 0.0715941783 \\
1 & 0.5593260180 & 0.5878499670 & 0.5314377478 & 0.0401564305 \\
2 & 0.6321692136 & 0.6171006698 & 0.5528987210 & 0.0214609733 \\
$\vdots$ & $\vdots$ & $\vdots$ & $\vdots$ & $\vdots$ \\
36 & 0.6065554098 & 0.6065554098 & 0.5452257174 & 0.0000000000 \\
\hline
\end{tabular}

Table 8: Modified Jungck Mann iteration scheme $(\beta=0.66)$

\begin{tabular}{c|c|c|c|c}
\hline$n$ & $T x_{\mathrm{n}}$ & $S x_{\mathrm{n}+1}$ & $x_{\mathrm{n}+1}$ & $\left|e_{\mathrm{n}}\right|$ \\
\hline 0 & 0.6931471806 & 0.6109141538 & 0.5484059872 & 0.0484059872 \\
1 & 0.6007394137 & 0.6065772237 & 0.5452416642 & 0.0031643231 \\
2 & 0.6065261621 & 0.6065554144 & 0.5452257208 & 0.0000159434 \\
3 & 0.6065554035 & 0.6065554098 & 0.5452257174 & 0.0000000034 \\
4 & 0.6065554098 & 0.6065554098 & 0.5452257174 & 0.0000000000 \\
\hline
\end{tabular}

Table 9: Jungck Ishikawa iteration scheme $(\beta=0.2879415656$ and $\alpha=0.86)$

\begin{tabular}{c|c|c|c|c}
\hline$n$ & $T x_{\mathrm{n}}$ & $S x_{\mathrm{n}+1}$ & $x_{\mathrm{n}+1}$ & $\left|e_{\mathrm{n}}\right|$ \\
\hline 0 & 0.6931471806 & 0.6192644038 & 0.5544642057 & 0.0544642057 \\
1 & 0.5897530267 & 0.6035011554 & 0.5429899033 & 0.0114743024 \\
2 & 0.6106645535 & 0.6072725030 & 0.5457497763 & 0.0027598730 \\
$\vdots$ & $\vdots$ & $\vdots$ & $\vdots$ & $\vdots$ \\
15 & 0.6065554098 & 0.6065554098 & 0.5452257174 & 0.0000000000 \\
\hline
\end{tabular}

Table 10: Modified Jungck Ishikawa iteration scheme $(\beta=0.2879415656$ and $\alpha=0.86)$

\begin{tabular}{c|c|c|c|c}
\hline$n$ & $T x_{\mathrm{n}}$ & $S x_{\mathrm{n}+1}$ & $x_{\mathrm{n}+1}$ & $\left|e_{\mathrm{n}}\right|$ \\
\hline 0 & 0.6931471806 & 0.6065554098 & 0.5452257174 & 0.0452257174 \\
1 & 0.6065554098 & 0.6065554098 & 0.5452257174 & 0.0000000000 \\
\hline
\end{tabular}

Example 4.3. Consider the nonlinear equation $e^{x}-\tan (x)=0$. Let us take $T x=e^{x}$ and $S x=\tan (x)$. The true graphical solution of given equation is $1.3063269404(10 D)$. If we choose initial guess $x_{0}=1.5$, then the comparision tables correct upto ten decimal places of Jungck, Jungck Mann, Jungck Ishikawa, modified Jungck Mann and modified Jungck Ishikawa iteration schemes are shown below. From tables, we can see that Jungck iteration scheme evaluate the solution of given equation in 19 iterations, Jungck Mann iterstion scheme $\beta=0.9757$ evaluate the solution in 19 iterations, modified 
Jungck Mann iterative scheme for $\beta=0.9757$ evaluate the solution in 6 iterations, Jungck Ishikawa iteration scheme for $\beta=0.27$ and $\alpha=0.98015$ evaluate the solution in 17 iterations and modified Jungck Ishikawa iterative scheme for $\beta=0.27$ and $\alpha=0.98015$ evaluate the solution in 5 iterations of the given equation (see Tables 11-15).

Table 11: Jungck iteration scheme

\begin{tabular}{c|c|c|c|c}
\hline$n$ & $T x_{\mathrm{n}}$ & $S x_{\mathrm{n}+1}$ & $x_{\mathrm{n}+1}$ & $\left|e_{\mathrm{n}}\right|$ \\
\hline 0 & 4.4816890703 & 4.4816890703 & 1.3512623360 & 0.1487376640 \\
1 & 3.8622979725 & 3.8622979725 & 1.3174465040 & 0.0338158320 \\
2 & 3.7338747596 & 3.7338747596 & 1.3091190649 & 0.0083274391 \\
3 & 3.7029102514 & 3.7029102514 & 1.3070305691 & 0.0020884958 \\
$\vdots$ & $\vdots$ & $\vdots$ & $\vdots$ & $\vdots$ \\
17 & 3.6925856857 & 3.6925856857 & 1.3063269404 & 0.0000000001 \\
18 & 3.6925856854 & 3.6925856854 & 1.3063269404 & 0.0000000000 \\
\hline
\end{tabular}

Table 12: Jungck Mann iteration scheme $(\beta=0.9757)$

\begin{tabular}{c|c|c|c|c}
\hline$n$ & $T x_{\mathrm{n}}$ & $S x_{\mathrm{n}+1}$ & $x_{\mathrm{n}+1}$ & $\left|e_{\mathrm{n}}\right|$ \\
\hline 0 & 4.4816890703 & 4.7154485306 & 1.3618234407 & 0.1381765593 \\
1 & 3.9033042605 & 3.9230393663 & 1.3212071100 & 0.0406163307 \\
2 & 3.7479428270 & 3.7521976729 & 1.3103397589 & 0.0108673511 \\
$\vdots$ & $\vdots$ & $\vdots$ & $\vdots$ & $\vdots$ \\
18 & 3.6925856854 & 3.6925856854 & 1.3063269404 & 0.0000000000 \\
\hline
\end{tabular}

Table 13: Modified Jungck Mann iteration scheme $(\beta=0.9757)$

\begin{tabular}{c|c|c|c|c}
\hline$n$ & $T x_{\mathrm{n}}$ & $S x_{\mathrm{n}+1}$ & $x_{\mathrm{n}+1}$ & $\left|e_{\mathrm{n}}\right|$ \\
0 & 4.4816890703 & 2.0196387593 & 1.1110458360 & 0.3889541640 \\
\hline 1 & 3.0375334954 & 3.5002310790 & 1.2925141066 & 0.1814682707 \\
2 & 3.6419312590 & 3.6908196870 & 1.3062062187 & 0.0136921121 \\
3 & 3.6921399371 & 3.6925863471 & 1.3063269856 & 0.0001207669 \\
4 & 3.6925858523 & 3.6925856853 & 1.3063269404 & 0.0000000452 \\
5 & 3.6925856854 & 3.6925856854 & 1.3063269404 & 0.0000000000 \\
\hline
\end{tabular}

Table 14: Jungck Ishikawa iteration scheme $(\beta=0.27$ and $\alpha=0.98015)$

\begin{tabular}{c|c|c|c|c}
\hline$n$ & $T x_{\mathrm{n}}$ & $S x_{\mathrm{n}+1}$ & $x_{\mathrm{n}+1}$ & $\left|e_{\mathrm{n}}\right|$ \\
\hline 0 & 4.4816890703 & 4.6032513462 & 1.3568822657 & 0.1431177343 \\
1 & 3.8840649225 & 3.8637833045 & 1.3175397857 & 0.0393424800 \\
2 & 3.7342230781 & 3.7286964189 & 1.3087720490 & 0.0087677367 \\
$\vdots$ & $\vdots$ & $\vdots$ & $\vdots$ & $\vdots$ \\
16 & 3.6925856855 & 3.6925856855 & 1.3063269404 & 0.0000000000 \\
\hline
\end{tabular}


Table 15: Modified Jungck Ishikawa iteration scheme $(\beta=0.27$ and $\alpha=0.98015)$

\begin{tabular}{c|c|c|c|c}
\hline$n$ & $T x_{\mathrm{n}}$ & $S x_{\mathrm{n}+1}$ & $x_{\mathrm{n}+1}$ & $\left|e_{\mathrm{n}}\right|$ \\
\hline 0 & 4.4816890703 & 2.5649928655 & 1.1990577549 & 0.3009422451 \\
1 & 3.3170157877 & 3.6430207157 & 1.3028973663 & 0.1038396113 \\
2 & 3.6799433803 & 3.6925103388 & 1.3063217920 & 0.0034244257 \\
3 & 3.6925666745 & 3.6925856858 & 1.3063269404 & 0.0000051484 \\
4 & 3.6925856855 & 3.6925856855 & 1.3063269404 & 0.0000000000 \\
\hline
\end{tabular}

Example 4.4. Consider the nonlinear equation $1-x-\sin (x)=0$. Let us take $T x=1-x$ and $S x=\sin (x)$. The true graphical solution of given equation is $0.5109734294(10 D)$. If we choose initial guess $x_{0}=0.5$, then the comparision tables correct upto ten decimal places of Jungck,Jungck Mann, modified Jungck Mann, Jungck Ishikawa and modified Jungck Ishikawa iteration schemes are shown below. From tables, we can see that Jungck iteration scheme evaluate the solution of given equation in 147 iterations, Jungck Mann Iteration scheme for $\beta=0.931$ diverged, modified Jungck Mann Iteration scheme for $\beta=0.931$ evaluate the solution in 4 iterations, Jungck Ishikawa iteration scheme for $\alpha=$ 0.945625 and $\beta=0.1$ evaluate the solution in 86 iterations and modified Jungck Ishikawa iteration scheme for $\alpha=0.945625$ and $\beta=0.1$ evaluate the solution in 3 iterations of the given equation (see Tables 15-20).

Table 16: Jungck iteration scheme

\begin{tabular}{c|c|c|c|c}
\hline$n$ & $T x_{\mathrm{n}}$ & $S x_{\mathrm{n}+1}$ & $x_{\mathrm{n}+1}$ & $\left|e_{\mathrm{n}}\right|$ \\
\hline 0 & 0.4794255386 & 0.4794255386 & 0.5205744614 & 0.0205744614 \\
1 & 0.4973785844 & 0.4973785844 & 0.5026214156 & 0.0179530458 \\
2 & 0.4817243973 & 0.4817243973 & 0.5182756027 & 0.0156541872 \\
3 & 0.4953829348 & 0.4953829348 & 0.5046170652 & 0.0136585375 \\
4 & 0.4834722701 & 0.4834722701 & 0.5165277299 & 0.0119106647 \\
5 & 0.4938638460 & 0.4938638460 & 0.5061361540 & 0.0103915759 \\
$\vdots$ & $\vdots$ & $\vdots$ & $\vdots$ & $\vdots$ \\
145 & 0.4890265706 & 0.4890265706 & 0.5109734294 & 0.0000000001 \\
146 & 0.4890265706 & 0.4890265706 & 0.5109734294 & 0.0000000000 \\
\hline
\end{tabular}

Table 17: Jungck Mann iteration scheme $(\beta=0.931)$

\begin{tabular}{c|c|c|c|c}
\hline$n$ & $T x_{\mathrm{n}}$ & $S x_{\mathrm{n}+1}$ & $x_{\mathrm{n}+1}$ & $\left|e_{\mathrm{n}}\right|$ \\
\hline 0 & 0.5000000000 & 0.4985803622 & 0.5219602933 & 0.0219602933 \\
1 & 0.4780397067 & 0.4794570119 & 0.5000358640 & 0.0219244292 \\
2 & 0.4999641360 & 0.4985491444 & 0.5219242805 & 0.0218884165 \\
$\vdots$ & $\vdots$ & $\vdots$ & $\vdots$ & $\vdots$ \\
\hline
\end{tabular}


Table 18: Modified Jungck Mann iteration scheme $(\beta=0.931)$

\begin{tabular}{c|c|c|c|c}
\hline$n$ & $T x_{\mathrm{n}}$ & $S x_{\mathrm{n}+1}$ & $x_{\mathrm{n}+1}$ & $\left|e_{\mathrm{n}}\right|$ \\
\hline 0 & 0.5000000000 & 0.4890029504 & 0.5109463505 & 0.0109463505 \\
1 & 0.4890536495 & 0.4890265508 & 0.5109734067 & 0.0000270562 \\
2 & 0.4890265933 & 0.4890265706 & 0.5109734294 & 0.0000000227 \\
3 & 0.4890265706 & 0.4890265706 & 0.5109734294 & 0.0000000000 \\
\hline
\end{tabular}

Table 19: Jungck Ishikawa iteration scheme $(\alpha=0.945625$ and $\beta=0.1$ )

\begin{tabular}{c|c|c|c|c}
\hline$n$ & $T x_{\mathrm{n}}$ & $S x_{\mathrm{n}+1}$ & $x_{\mathrm{n}+1}$ & $\left|e_{\mathrm{n}}\right|$ \\
\hline 0 & 0.5000000000 & 0.4966628720 & 0.5197496595 & 0.0197496595 \\
1 & 0.4802503405 & 0.4829299528 & 0.5039976346 & 0.0157520249 \\
2 & 0.4960023654 & 0.4938792797 & 0.5165454793 & 0.0125478447 \\
$\vdots$ & $\vdots$ & $\vdots$ & $\vdots$ & $\vdots$ \\
85 & 0.4890265706 & 0.4890265706 & 0.5109734294 & 0.0000000000 \\
\hline
\end{tabular}

Table 20: Modified Jungck Ishikawa iteration scheme $(\alpha=0.945625$ and $\beta=0.1)$

\begin{tabular}{c|c|c|c|c}
\hline$n$ & $T x_{\mathrm{n}}$ & $S x_{\mathrm{n}+1}$ & $x_{\mathrm{n}+1}$ & $\left|e_{\mathrm{n}}\right|$ \\
\hline 0 & 0.5000000000 & 0.4890169916 & 0.5109624477 & 0.0109624477 \\
1 & 0.4890375523 & 0.4890265706 & 0.5109734294 & 0.0000109817 \\
2 & 0.4890265706 & 0.4890265706 & 0.5109734294 & 0.0000000000 \\
\hline
\end{tabular}

Example 4.5. Consider the nonlinear equation $e^{x^{2}}-\frac{5}{e^{2 x}}=0$. Let us take $T x=-e^{x^{2}}$ and $S x=-\frac{5}{e^{2 x}}$. The true graphical solution of given equation is $0.6153754710(10 D)$. If we choose initial guess $x_{0}=0.5$, then the comparision tables correct upto ten decimal places of Jungck, Jungck Mann and Jungck Ishikawa, modified Jungck Mann and modified Jungck Ishikawa iteration schemes are shown below.From tables, we can see that Jungck iteration scheme evaluate the solution of given equation in 47 iterations, Jungck Mann iteration scheme for $\beta=0.96581$ evaluate the solution in 40 iterations, modified Jungck Mann iteration scheme for $\beta=0.96581$ evaluate the solution in 5 iterations, Jungck Ishikawa iteration scheme for $\alpha=0.96988718$ and $\beta=0.1$ evaluate the solution in 31 iterations and modified Jungck Ishikawa iteration scheme for $\alpha=0.96988718$ and $\beta=0.1$ evaluate the solution in 4 iterations of 
the given equcation (see Tables 21-25).

Table 21: Jungck iteration scheme

\begin{tabular}{c|c|c|c|c}
\hline$n$ & $T x_{\mathrm{n}}$ & $S x_{\mathrm{n}+1}$ & $x_{\mathrm{n}+1}$ & $\left|e_{\mathrm{n}}\right|$ \\
\hline 0 & 1.2840254167 & 1.2840254167 & 0.6797189562 & 0.1797189562 \\
1 & 1.5872736508 & 1.5872736508 & 0.5737100265 & 0.1060089297 \\
2 & 1.3897768497 & 1.3897768497 & 0.6401473590 & 0.0664373325 \\
3 & 1.5064993396 & 1.5064993396 & 0.5998246356 & 0.0403227233 \\
4 & 1.4330278645 & 1.4330278645 & 0.6248241595 & 0.0249995238 \\
5 & 1.4775794325 & 1.4775794325 & 0.6095163411 & 0.0153078184 \\
$\vdots$ & $\vdots$ & $\vdots$ & $\vdots$ & $\vdots$ \\
41 & 1.4603658268 & 1.4603658268 & 0.6153754709 & 0.0000000004 \\
42 & 1.4603658261 & 1.4603658261 & 0.615375471 & 0.0000000002 \\
43 & 1.4603658266 & 1.4603658266 & 0.6153754710 & 0.0000000001 \\
44 & 1.4603658263 & 1.4603658263 & 0.6153754711 & 0.0000000001 \\
45 & 1.4603658265 & 1.4603658265 & 0.6153754710 & 0.0000000001 \\
46 & 1.4603658264 & 1.4603658264 & 0.6153754711 & 0.000000000 \\
\hline
\end{tabular}

Table 22: Jungck Mann iteration scheme $(\beta=0.96581)$

\begin{tabular}{c|c|c|c|c}
\hline$n$ & $T x_{\mathrm{n}}$ & $S x_{\mathrm{n}+1}$ & $x_{\mathrm{n}+1}$ & $\left|e_{\mathrm{n}}\right|$ \\
\hline 0 & -1.2840254167 & -1.3030135782 & 0.6723790968 & 0.1723790968 \\
1 & -1.5715991217 & -1.5624161820 & 0.5816022274 & 0.0907768694 \\
2 & -1.4025067215 & -1.4079740259 & 0.6336430512 & 0.0520408238 \\
$\vdots$ & $\vdots$ & $\vdots$ & $\vdots$ & $\vdots$ \\
39 & -1.4603658264 & -1.4603658264 & 0.6153754710 & 0.0000000000 \\
\hline
\end{tabular}

Table 23: Modified Jungck Mann iteration scheme $(\beta=0.96581)$

\begin{tabular}{c|c|c|c|c}
\hline$n$ & $T x_{\mathrm{n}}$ & $S x_{\mathrm{n}+1}$ & $x_{\mathrm{n}+1}$ & $\left|e_{\mathrm{n}}\right|$ \\
\hline 0 & -1.2840254167 & -1.5378549349 & 0.5895246832 & 0.0895246832 \\
1 & -1.4155800156 & -1.4640066147 & 0.6141304894 & 0.0246058062 \\
2 & -1.4581321363 & -1.4603658394 & 0.6153754666 & 0.0012449772 \\
3 & -1.4603658184 & -1.4603658263 & 0.6153754710 & 0.0000000044 \\
4 & -1.4603658264 & -1.4603658264 & 0.6153754710 & 0.0000000000 \\
\hline
\end{tabular}

Table 24: Jungck Ishikawa iteration scheme $(\alpha=0.96988718$ and $\beta=0.1)$

\begin{tabular}{c|c|c|c|c}
\hline$n$ & $T x_{\mathrm{n}}$ & $S x_{\mathrm{n}+1}$ & $x_{\mathrm{n}+1}$ & $\left|e_{\mathrm{n}}\right|$ \\
\hline 0 & -1.2840254167 & -1.3202838247 & 0.6657955901 & 0.1657955901 \\
1 & -1.5578143292 & -1.5329492512 & 0.5911222087 & 0.0746733815 \\
2 & -1.4182524796 & -1.4278460624 & 0.6266354269 & 0.0355132182 \\
$\vdots$ & $\vdots$ & $\vdots$ & $\vdots$ & $\vdots$ \\
30 & -1.4603658264 & -1.4603658264 & 0.6153754710 & 0.0000000000 \\
\hline
\end{tabular}


Table 25: Modified Jungck Ishikawa iteration scheme $(\alpha=0.96988718$ and $\beta=0.1)$

\begin{tabular}{c|c|c|c|c}
\hline$n$ & $T x_{\mathrm{n}}$ & $S x_{\mathrm{n}+1}$ & $x_{\mathrm{n}+1}$ & $\left|e_{\mathrm{n}}\right|$ \\
\hline 0 & -1.2840254167 & -1.5187560057 & 0.5957731650 & 0.0957731650 \\
1 & -1.4261031636 & -1.4618889957 & 0.6148542402 & 0.0190810752 \\
2 & -1.4594296907 & -1.4603658264 & 0.6153754710 & 0.0005212309 \\
3 & -1.4603658264 & -1.4603658264 & 0.6153754710 & 0.0000000000 \\
\hline
\end{tabular}

Example 4.6. Consider the nonlinear equation $\tan (x)-\frac{1}{2 x}=0$. Let us take $T x=2 \tan (x)$ and $S x=\frac{1}{x}$. The true graphical solution of given equation is $0.6532711871(10 D)$. If we choose initial guess $x_{0}=0.7$, then the comparision tables correct upto ten decimal places of Jungck, Jungck Mann, Jungck Ishikawa, modified Jungck Mann and modified Jungck Ishikawa iteration schemes are shown below. From tables, we can see that Jungck iteration scheme evaluate the solution of given equation in 71 iterations, Jungck Mann Iteration scheme for $\beta=0.82$ evaluate the solution in 27 iterations, modified Jungck Mann Iterative scheme for $\beta=0.82$ evaluate the solution in 5 iterations, Jungck Ishikawa iteration scheme for $\beta=0.28289$ and $\alpha=0.9$ evaluate the solution in 31 iteration and modified Jungck Ishikawa iteration scheme for $\beta=0.28289$ and $\alpha=0.9$ evaluate the solution in 4 iteration of the given equation (see Tables 26-30).

Table 26: Jungck iteration scheme

\begin{tabular}{c|c|c|c|c}
\hline$n$ & $T x_{\mathrm{n}}$ & $S x_{\mathrm{n}+1}$ & $x_{\mathrm{n}+1}$ & $\left|e_{\mathrm{n}}\right|$ \\
\hline 0 & 1.4285714286 & 1.4285714286 & 0.6202494860 & 0.0797505140 \\
1 & 1.6122544598 & 1.6122544598 & 0.6784659072 & 0.0582164212 \\
2 & 1.4739134117 & 1.4739134117 & 0.6351010057 & 0.0433649016 \\
3 & 1.5745526949 & 1.5745526949 & 0.6669343221 & 0.0318333164 \\
$\vdots$ & $\vdots$ & $\vdots$ & $\vdots$ & $\vdots$ \\
69 & 1.5307578534 & 1.5307578534 & 0.6532711871 & 0.0000000001 \\
70 & 1.5307578533 & 1.5307578533 & 0.6532711871 & 0.0000000000 \\
\hline
\end{tabular}

Table 27: Jungck Mann iteration scheme $(\beta=0.82)$

\begin{tabular}{c|c|c|c|c}
\hline$n$ & $T x_{\mathrm{n}}$ & $S x_{\mathrm{n}+1}$ & $x_{\mathrm{n}+1}$ & $\left|e_{\mathrm{n}}\right|$ \\
\hline 0 & 1.4285714286 & 1.4746523884 & 0.6353404081 & 0.0646595919 \\
1 & 1.5739593881 & 1.5560841282 & 0.6612078185 & 0.0258674103 \\
2 & 1.5123838105 & 1.5202498677 & 0.6499496370 & 0.0112581815 \\
$\vdots$ & $\vdots$ & $\vdots$ & $\vdots$ & $\vdots$ \\
26 & 1.5307578534 & 1.5307578534 & 0.6532711871 & 0.0000000000 \\
\hline
\end{tabular}


Table 28: Modified Jungck Mann iteration scheme $(\beta=0.82)$

\begin{tabular}{c|c|c|c|c}
\hline$n$ & $T x_{\mathrm{n}}$ & $S x_{\mathrm{n}+1}$ & $x_{\mathrm{n}+1}$ & $\left|e_{\mathrm{n}}\right|$ \\
\hline 0 & 1.4285714286 & 1.5436879204 & 0.6573352986 & 0.0426647014 \\
1 & 1.5212936262 & 1.5308651753 & 0.6533050245 & 0.0040302740 \\
2 & 1.5306785689 & 1.5307579308 & 0.6532712115 & 0.0000338130 \\
3 & 1.5307577961 & 1.5307578533 & 0.6532711871 & 0.0000000244 \\
4 & 1.5307578534 & 1.5307578534 & 0.6532711871 & 0.0000000000 \\
\hline
\end{tabular}

Table 29: Jungck Ishikawa iteration scheme $(\alpha=0.9$ and $\beta=0.28289)$

\begin{tabular}{c|c|c|c|c}
\hline$n$ & $T x_{\mathrm{n}}$ & $S x_{\mathrm{n}+1}$ & $x_{\mathrm{n}+1}$ & $\left|e_{\mathrm{n}}\right|$ \\
\hline 0 & 1.4285714286 & 1.4950384397 & 0.6419115180 & 0.0580884820 \\
1 & 1.5578471051 & 1.5392794188 & 0.6559524933 & 0.0140409753 \\
2 & 1.5245006464 & 1.5287327278 & 0.6526323584 & 0.0033201349 \\
$\vdots$ & $\vdots$ & $\vdots$ & $\vdots$ & $\vdots$ \\
30 & 1.5307578533 & 1.5307578533 & 0.6532711871 & 0.0000000000 \\
\hline
\end{tabular}

Table 30: Modified Jungck Ishikawa iteration scheme $(\alpha=0.9$ and $\beta=0.28289)$

\begin{tabular}{c|c|c|c|c}
\hline$n$ & $T x_{\mathrm{n}}$ & $S x_{\mathrm{n}+1}$ & $x_{\mathrm{n}+1}$ & $\left|e_{\mathrm{n}}\right|$ \\
\hline 0 & 1.4285714286 & 1.5342361319 & 0.6543669587 & 0.0456330413 \\
1 & 1.5281945194 & 1.5307594849 & 0.6532717015 & 0.0010952572 \\
2 & 1.5307566479 & 1.5307578533 & 0.6532711871 & 0.0000005144 \\
3 & 1.5307578533 & 1.5307578533 & 0.6532711871 & 0.0000000000 \\
\hline
\end{tabular}

Example 4.7. Consider the nonlinear equation $2 x+3 \cos (x)-e^{x}=0$. Let us take $T x=e^{x}-3 \cos (x)$ and $S x=2 x$. The true graphical solution of given equation is $-0.8109753311(10 D)$. If we choose initial guess $x_{0}=-0.9$, then the comparision tables correct upto ten decimal places of Jungck, Jungck Mann , modified Jungck Mann, Jungck Ishikawa and modified Jungck Ishikawa iterations are shown below. From tables, we can see that Jungck iteration scheme evaluate the solution of given equation in 153 iterations, Jungck Mann Iterative scheme for $\beta=0.8459$ evaluate the solution in 41 iterations, modified Jungck Mann Iterative scheme for $\beta=0.8459$ evaluate the solution in 5 iterations, Jungck Ishikawa iterative scheme for $\alpha=0.959$ and $\beta=0.41026$ evaluate the solution in 13 iterations and modified Jungck Ishikawa iterative scheme for $\alpha=0.959$ and $\beta=0.41026$ evaluate the solution in 3 iterations of the given 
equcation (see Tables 31-35).

Table 31: Jungck iteration scheme

\begin{tabular}{c|c|c|c|c}
\hline$n$ & $T x_{\mathrm{n}}$ & $S x_{\mathrm{n}+1}$ & $x_{\mathrm{n}+1}$ & $\left|e_{\mathrm{n}}\right|$ \\
\hline 0 & -1.4582602451 & -1.4582602451 & -0.7291301225 & 0.1708698775 \\
1 & -1.7549342714 & -1.7549342714 & -0.8774671357 & 0.1483370132 \\
2 & -1.5014689938 & -1.5014689938 & -0.7507344969 & 0.1267326388 \\
3 & -1.7215443016 & -1.7215443016 & -0.8607721508 & 0.1100376539 \\
$\vdots$ & $\vdots$ & $\vdots$ & $\vdots$ & $\vdots$ \\
151 & -1.6219506622 & -1.6219506622 & -0.8109753311 & 0.0000000001 \\
152 & -1.6219506621 & -1.6219506621 & -0.8109753311 & 0.0000000000 \\
\hline
\end{tabular}

Table 32: Jungck Mann iteration scheme $(\beta=0.8459)$

\begin{tabular}{c|c|c|c|c}
\hline$n$ & $T x_{\mathrm{n}}$ & $S x_{\mathrm{n}+1}$ & $x_{\mathrm{n}+1}$ & $\left|e_{\mathrm{n}}\right|$ \\
\hline 0 & -1.4582602451 & -1.5109223413 & -0.7554611707 & 0.1445388293 \\
1 & -1.7140723826 & -1.6827669612 & -0.8413834806 & 0.0859223100 \\
2 & -1.5681822910 & -1.5858397887 & -0.7929198943 & 0.0484635863 \\
$\vdots$ & $\vdots$ & $\vdots$ & $\vdots$ & $\vdots$ \\
40 & -1.6219506621 & -1.6219506621 & -0.8109753311 & 0.0000000000 \\
\hline
\end{tabular}

Table 33: Modified Jungck Mann iteration scheme $(\beta=0.8459)$

\begin{tabular}{c|c|c|c|c}
\hline$n$ & $T x_{\mathrm{n}}$ & $S x_{\mathrm{n}+1}$ & $x_{\mathrm{n}+1}$ & $\left|e_{\mathrm{n}}\right|$ \\
\hline 0 & -1.4582602451 & -1.6091341275 & -0.8045670638 & 0.0954329362 \\
1 & -1.6329882023 & -1.6218782962 & -0.8109391481 & 0.0063720843 \\
2 & -1.6220132735 & -1.6219506558 & -0.8109753279 & 0.0000361798 \\
3 & -1.6219506676 & -1.6219506621 & -0.8109753311 & 0.0000000032 \\
4 & -1.6219506621 & -1.6219506621 & -0.8109753311 & 0.0000000000 \\
\hline
\end{tabular}

Table 34: Jungck Ishikawa iteration scheme $(\alpha=0.959$ and $\beta=0.41026)$

\begin{tabular}{c|c|c|c|c}
\hline$n$ & $T x_{\mathrm{n}}$ & $S x_{\mathrm{n}+1}$ & $x_{\mathrm{n}+1}$ & $\left|e_{\mathrm{n}}\right|$ \\
\hline 0 & -1.4582602451 & -1.5974186041 & -0.7987093021 & 1.6987093021 \\
1 & -1.6429867699 & -1.6257780647 & -0.8128890324 & 0.0141797303 \\
2 & -1.6186344964 & -1.6213633194 & -0.8106816597 & 0.0022073727 \\
$\vdots$ & $\vdots$ & $\vdots$ & $\vdots$ & $\vdots$ \\
12 & -1.6219506621 & -1.6219506621 & -0.8109753311 & 0.0000000000 \\
\hline
\end{tabular}


Table 35: Modified Jungck Ishikawa iteration scheme $(\alpha=0.959$ and $\beta=0.41026)$

\begin{tabular}{c|c|c|c|c|}
\hline$n$ & $T x_{\mathrm{n}}$ & $S x_{\mathrm{n}+1}$ & $x_{\mathrm{n}+1}$ & $\left|e_{\mathrm{n}}\right|$ \\
\hline 0 & -1.4582602451 & -1.6219470030 & -0.8109735015 & 0.0890264985 \\
1 & -1.6219538281 & -1.6219506621 & -0.8109753311 & 0.0000018296 \\
2 & -1.6219506621 & -1.6219506621 & -0.8109753311 & 0.0000000000 \\
\hline
\end{tabular}

Example 4.8. Consider the nonlinear equation $4 \sin ^{-1}(x)-4 x \sqrt{\left(1-x^{2}\right)}$ $+8 x^{2} \cos ^{-1}(x)-\pi=0$. Let us take $T x=4 x \sqrt{\left(1-x^{2}\right)}-8 x^{2} \cos ^{-1}(x)+\pi, S x=$ $4 \sin ^{-1}(x)$ and $S x=4 \sin ^{-1}(x)$. The true graphical solution of given equation is $0.5793642365(10 D)$. If we choose initial guess $x_{0}=0.52$, then the comparision tables correct upto ten decimal places of Jungck, Jungck Mann, modified Jungck Mann, Jungck Ishikawa and modified Jungck Ishikawa iterationschemes are shown below. From tables, we can see that Jungck iteration scheme evaluate the solution of given equation in 77 iterations, Jungck Mann iteration scheme for $\beta=0.6950029203$ evaluate the solution in 17 iterations, modified Jungck Mann iteration scheme for $\beta=0.6950029203$ evaluate the solution in 2 iterations, Jungck Ishikawa iteration scheme for $\alpha=0.891$ and $\beta=0.3991341$ evaluate the solution in 11 iterations and modified Jungck Ishikawa iteration scheme for $\alpha=0.891$ and $\beta=0.3991341$ evaluate the solution in 3 iterations of the given equation (see Tables 36-40).

Table 36: Jungck iteration scheme

\begin{tabular}{c|c|c|c|c}
\hline$n$ & $T x_{\mathrm{n}}$ & $S x_{\mathrm{n}+1}$ & $x_{\mathrm{n}+1}$ & $\left|e_{\mathrm{n}}\right|$ \\
\hline 0 & 2.70325983 & 2.703259837 & 0.62553335 & 0.10553335 \\
1 & 2.29215848 & 2.29215848 & 2.70325984 & 0.08334474 \\
2 & 2.61743109 & 2.61743109 & 0.60864980 & 0.06646119 \\
3 & 2.35730769 & 2.35730769 & 2.61743109 & 0.05284819 \\
4 & 2.56424910 & 2.56424910 & 0.59804715 & 0.04224554 \\
5 & 2.39860595 & 2.39860595 & 0.56435480 & 0.03369235 \\
$\vdots$ & $\vdots$ & $\vdots$ & $\vdots$ & $\vdots$ \\
75 & 2.47179384 & 2.47179384 & 0.57936423 & 0.00000001 \\
76 & 2.47179386 & 2.47179386 & 0.57936424 & 0.00000000 \\
\hline
\end{tabular}


Table 37: Jungck Mann iteration scheme $(\beta=0.6950029203)$

\begin{tabular}{c|c|c|c|c}
\hline$n$ & $T x_{\mathrm{n}}$ & $S x_{\mathrm{n}+1}$ & $x_{\mathrm{n}+1}$ & $\left|e_{\mathrm{n}}\right|$ \\
\hline 0 & 2.7032598372 & 2.5459252531 & 0.5943694265 & 0.0743694265 \\
1 & 2.4129803137 & 2.4535281320 & 0.5756362552 & 0.0187331713 \\
2 & 2.4864290717 & 2.4763943812 & 0.5803012907 & 0.0046650355 \\
$\vdots$ & $\vdots$ & $\vdots$ & $\vdots$ & $\vdots$ \\
16 & 2.4717938486 & 2.4717938486 & 0.5793642365 & 0.0000000000 \\
\hline
\end{tabular}

Table 38: Modified Jungck Mann iteration scheme $(\beta=0.6950029203)$

\begin{tabular}{c|c|c|c|c}
\hline$n$ & $T x_{\mathrm{n}}$ & $S x_{\mathrm{n}+1}$ & $x_{\mathrm{n}+1}$ & $\left|e_{\mathrm{n}}\right|$ \\
\hline 0 & 2.7032598372 & 2.4717938486 & 0.5793642365 & 0.0593642365 \\
1 & 2.4717938486 & 2.4717938486 & 0.5793642365 & 0.0000000000 \\
\hline
\end{tabular}

Table 39: Jungck Ishikawa iteration scheme $(\alpha=0.891$ and $\beta=0.3991341)$

\begin{tabular}{c|c|c|c|c}
\hline$n$ & $T x_{\mathrm{n}}$ & $S x_{\mathrm{n}+1}$ & $x_{\mathrm{n}+1}$ & $\left|e_{\mathrm{n}}\right|$ \\
\hline 0 & 2.7032598372 & 2.4971256681 & 0.5845143776 & 0.0645143776 \\
1 & 2.4515855985 & 2.4694595702 & 0.5788884885 & 0.0000644928 \\
2 & 2.4736612871 & 2.4720078118 & 0.5794078344 & 0.0005193458 \\
$\vdots$ & $\vdots$ & $\vdots$ & $\vdots$ & $\vdots$ \\
10 & 2.4717938486 & 2.4717938486 & 0.5793642365 & 0.0000000000 \\
\hline
\end{tabular}

Table 40: Modified Jungck Ishikawa iteration scheme ( $\alpha=0.891$ and $\beta=0.3991341)$

\begin{tabular}{c|c|c|c|c}
\hline$n$ & $T x_{\mathrm{n}}$ & $S x_{\mathrm{n}+1}$ & $x_{\mathrm{n}+1}$ & $\left|e_{\mathrm{n}}\right|$ \\
\hline 0 & 2.7032598372 & 2.4714773552 & 0.5792997437 & 0.0592997437 \\
1 & 2.4720469948 & 2.4717938485 & 0.5793642365 & 0.0000644928 \\
2 & 2.4717938486 & 2.4717938486 & 0.5793642365 & 0.0000000000 \\
\hline
\end{tabular}

Example 4.9. Consider the nonlinear equation $e^{x}-2-\cos \left(e^{x}-2\right)=0$. Let us take $T x=\cos \left(e^{x}-2\right)$ and $S x=e^{x}-2$. The true graphical solution of given equation is $1.0076239716(10 D)$. If we choose initial guess $x_{0}=1.5$, then the comparision tables correct upto ten decimal places of Jungck, Jungck Mann, modified Jungck Mann, Jungck Ishikawa and modified Jungck Ishikawa iteration schemes are shown below. From tables, we can see that Jungck iteration scheme evaluate the solution of given equation in 50 iterations, Jungck Mann iteration scheme for $\beta=0.92$ evaluate the solution in 35 iterations, modified Jungck Mann iteration scheme for $\beta=0.92$ evaluate the solution in 8 
iterations, Jungck Ishikawa iteration scheme for $\alpha=0.9337912$ and $\beta=0.1$ evaluate the solution in 31 iterations and modified Jungck Ishikawa iteration scheme for $\alpha=0.9337912$ and $\beta=0.1$ evaluate the solution in 6 iterations of the given equation (see Tables 41-45).

Table 41: Jungck iteration scheme

\begin{tabular}{c|c|c|c|c}
\hline$n$ & $T x_{\mathrm{n}}$ & $S x_{\mathrm{n}+1}$ & $x_{\mathrm{n}+1}$ & $\left|e_{\mathrm{n}}\right|$ \\
\hline 0 & 0.7529375549 & 0.7529375549 & 1.0126685433 & 0.0126685433 \\
1 & 0.7296833636 & 0.7296833636 & 1.0041856184 & 0.0084829249 \\
2 & 0.7453855202 & 0.7453855202 & 1.0099215096 & 0.0057358912 \\
3 & 0.7348264759 & 0.7348264759 & 1.0060679881 & 0.0038535215 \\
4 & 0.7419471053 & 0.7419471053 & 1.0086682904 & 0.0026003023 \\
5 & 0.7371542502 & 0.7371542502 & 1.0069187859 & 0.0017495045 \\
$\vdots$ & $\vdots$ & $\vdots$ & $\vdots$ & $\vdots$ \\
48 & 0.7390851333 & 0.7390851333 & 1.0076239717 & 0.0000000001 \\
49 & 0.7390851332 & 0.7390851332 & 1.0076239716 & 0.0000000000 \\
\hline
\end{tabular}

Table 42: Jungck Mann iteration scheme $(\beta=0.92)$

\begin{tabular}{c|c|c|c|c}
\hline$n$ & $T x_{\mathrm{n}}$ & $S x_{\mathrm{n}+1}$ & $x_{\mathrm{n}+1}$ & $\left|e_{\mathrm{n}}\right|$ \\
\hline 0 & -0.7900513426 & -0.5283121095 & 0.3864099669 & 1.1135900331 \\
1 & 0.8636591260 & 0.7523014271 & 1.0124374443 & 0.6260274774 \\
2 & 0.7301181906 & 0.7318928495 & 1.0049947204 & 0.0074427239 \\
$\vdots$ & $\vdots$ & $\vdots$ & $\vdots$ & $\vdots$ \\
34 & 0.7390851332 & 0.7390851332 & 1.0076239717 & 0.0000000000 \\
\hline
\end{tabular}

Table 43: Modified Jungck Mann iteration scheme $(\beta=0.92)$

\begin{tabular}{c|c|c|c|c}
\hline$n$ & $T x_{\mathrm{n}}$ & $S x_{\mathrm{n}+1}$ & $x_{\mathrm{n}+1}$ & $\left|e_{\mathrm{n}}\right|$ \\
\hline 0 & -0.7900513425 & 0.2748994024 & 0.8219358327 & 0.6780641673 \\
1 & 0.9624525097 & 0.5163877552 & 0.9228244427 & 0.0691065510 \\
2 & 0.8696083667 & 0.6964362497 & 0.9919309936 & 0.0691065510 \\
3 & 0.7671331565 & 0.7376504595 & 1.0071000560 & 0.0151690624 \\
4 & 0.7400507857 & 0.7390815202 & 1.0076226526 & 0.0005225966 \\
5 & 0.7390875670 & 0.7390851279 & 1.0076239697 & 0.0000013171 \\
6 & 0.7390851368 & 0.7390851332 & 1.0076239717 & 0.0000000020 \\
7 & 0.7390851332 & 0.7390851332 & 1.0076239717 & 0.0000000000 \\
\hline
\end{tabular}


Table 44: Jungck Ishikawa iteration scheme $(\alpha=0.9337912$ and $\beta=0.1)$

\begin{tabular}{c|c|c|c|c}
\hline$n$ & $T x_{\mathrm{n}}$ & $S x_{\mathrm{n}+1}$ & $x_{\mathrm{n}+1}$ & $\left|e_{\mathrm{n}}\right|$ \\
0 & -0.7900513426 & -0.3503313386 & 0.5005744565 & 0.9994255435 \\
1 & 0.9392590460 & 0.8878089262 & 1.0604980576 & 0.5599236011 \\
2 & 0.6311131359 & 0.6665057921 & 0.9807689232 & 0.0797291343 \\
$\vdots$ & $\vdots$ & $\vdots$ & $\vdots$ & $\vdots$ \\
30 & 0.7390851332 & 0.7390851332 & 1.0076239717 & 0.0000000000 \\
\hline
\end{tabular}

Table 45: Modified Jungck Ishikawa iteration scheme $(\alpha=0.9337912$ and $\beta=0.1)$

\begin{tabular}{c|c|c|c|c}
\hline$n$ & $T x_{\mathrm{n}}$ & $S x_{\mathrm{n}+1}$ & $x_{\mathrm{n}+1}$ & $\left|e_{\mathrm{n}}\right|$ \\
\hline 0 & -0.7900513426 & 0.2153125014 & 0.7953934777 & 0.7046065223 \\
1 & 0.9769096751 & 0.5334802556 & 0.9295939526 & 0.1342004749 \\
2 & 0.8610424636 & 0.7142683653 & 0.9985224384 & 0.0689284858 \\
3 & 0.7555727115 & 0.7387680168 & 1.0075081904 & 0.0089857520 \\
4 & 0.7392987094 & 0.7390851332 & 1.0076239717 & 0.0001157813 \\
5 & 0.7390851332 & 0.7390851332 & 1.0076239717 & 0.0000000000 \\
\hline
\end{tabular}

Example 4.10. Consider the nonlinear equation $x^{3}+4 x^{2}-5 x-10=0$. Let us take $T x=x^{3}+4 x^{2}-10$ and $S x=5 x$. The true graphical solution of given equation is $(10 D)$. If we choose initial guess $x_{0}=-1$, then Jungck iteration cannot converges to the true solution. But the comparision tables correct upto ten decimal places of Jungck Mann, modified Jungck Mann, Jungck Ishikawa and modified Jungck Ishikawa iteration schemes are shown below.From tables, we can see that Jungck Mann Iteration scheme for $\beta=0.578841$ evaluate the solution in 15 iterations, modified Jungck Mann iteration scheme for $\beta=$ 0.578841 evaluate the solution in 4 iterations, Jungck Ishikawa iteration scheme for $\alpha=0.88063307794$ and $\beta=0.4$ evaluate the solution in 8 iterations and modified Jungck Ishikawa iteration scheme for $\alpha=0.88063307794$ and $\beta=0.4$ evaluate the solution in 2 iterations of the given equation (see Tables 46-50).

Table 46: Jungck iteration scheme

\begin{tabular}{c|c|c|c|c}
\hline$n$ & $T x_{\mathrm{n}}$ & $S x_{\mathrm{n}+1}$ & $x_{\mathrm{n}+1}$ & $\left|e_{\mathrm{n}}\right|$ \\
\hline 0 & -7.0000000000 & -7.0000000000 & -1.4000000000 & 2.4000000000 \\
1 & -4.9040000000 & -4.9040000000 & -0.9808000000 & 0.4192000000 \\
2 & -7.0956242821 & -7.0956242821 & -1.4191248564 & 0.4383248564 \\
$\vdots$ & $\vdots$ & $\vdots$ & $\vdots$ & $\vdots$ \\
\hline
\end{tabular}


Table 47: Jungck Mann iteration scheme $(\beta=0.578841)$

\begin{tabular}{c|c|c|c|c}
\hline$n$ & $T x_{\mathrm{n}}$ & $S x_{\mathrm{n}+1}$ & $x_{\mathrm{n}+1}$ & $\left|e_{\mathrm{n}}\right|$ \\
\hline 0 & -7.0000000000 & -6.1576820000 & -1.2315364000 & 0.2315364000 \\
1 & -6.0052195912 & -5.9512900794 & -1.1902580159 & 0.0412783841 \\
2 & -5.9844336354 & -5.9907141930 & -1.1981428386 & 0.0078848227 \\
$\vdots$ & $\vdots$ & $\vdots$ & $\vdots$ & $\vdots$ \\
14 & 5.9844338974 & -5.9844338974 & -1.1968867795 & 0.0000000000 \\
\hline
\end{tabular}

Table 48: Modified Jungck Mann iteration scheme $(\beta=0.578841)$

\begin{tabular}{c|c|c|c|c}
\hline$n$ & $T x_{\mathrm{n}}$ & $S x_{\mathrm{n}+1}$ & $x_{\mathrm{n}+1}$ & $\left|e_{\mathrm{n}}\right|$ \\
\hline 0 & -7.0000000000 & -5.9647350000 & -1.1929470000 & 0.1929470000 \\
1 & -6.0052195912 & -5.9844341456 & -1.1968868291 & 0.0039398291 \\
2 & -5.9844336354 & -5.9844338973 & -1.1968867795 & 0.0000000497 \\
3 & 5.9844338974 & -5.9844338974 & -1.1968867795 & 0.0000000000 \\
\hline
\end{tabular}

Table 49: Jungck Ishikawa iteration scheme $(\alpha=0.88063307794$ and $\beta=0.4)$

\begin{tabular}{c|c|c|c|c}
\hline$n$ & $T x_{\mathrm{n}}$ & $S x_{\mathrm{n}+1}$ & $x_{\mathrm{n}+1}$ & $\left|e_{\mathrm{n}}\right|$ \\
\hline 0 & -7.0000000000 & -6.0378225598 & -1.2075645120 & 2.2075645120 \\
1 & -5.9280369209 & -5.9819989431 & -1.1963997886 & 0.0111647233 \\
2 & -5.9870038848 & -5.9845456964 & -1.1969091393 & 0.0005093507 \\
$\vdots$ & $\vdots$ & $\vdots$ & $\vdots$ & $\vdots$ \\
8 & -5.9844338974 & -5.9844338974 & -1.1968867795 & 0.0000000000 \\
\hline
\end{tabular}

Table 50: Modified Jungck Ishikawa iteration scheme $(\alpha=0.88063307794$ and $\beta=0.4)$

\begin{tabular}{c|c|c|c|c}
\hline$n$ & $T x_{\mathrm{n}}$ & $S x_{\mathrm{n}+1}$ & $x_{\mathrm{n}+1}$ & $\left|e_{\mathrm{n}}\right|$ \\
\hline 0 & -7.0000000000 & -5.9844338974 & -1.1968867795 & 0.1968867795 \\
1 & -5.9844338974 & -5.9844338974 & -1.1968867795 & 0.0000000000 \\
\hline
\end{tabular}

Example 4.11. Consider the nonlinear equation $2 x-e^{\cos (x)}=0$. Let us take $T x=e^{\cos (x)}$ and $S x=2 x$. The true graphical solution of given equation is $0.9179048993(10 D)$. If we choose initial guess $x_{0}=1$, then the comparision tables correct upto ten decimal places of Jungck, Jungck Mann, modified Jungck Mann, Jungck Ishikawa and modified Jungck Ishikawa iteration schemes are shown below.From tables, we can see that Jungck iteration scheme evaluate the solution of given equation in 71 iterations, Jungck Mann iteration scheme 
for $\beta=0.98$ evaluate the solution in 59 iterations, modified Jungck Mann iteration scheme for $\beta=0.98$ evaluate the solution in 5 iterations, Jungck Ishikawa iteration scheme for $\alpha=0.9876$ and $\beta=0.3$ evaluate the solution in 21 iterations and modified Jungck Ishikawa iterative scheme for $\alpha=0.9876$ and $\beta=0.3$ evaluate the solution in 4 iterations of the given equation (see Tables $51-55)$.

Table 51: Jungck iteration scheme

\begin{tabular}{c|c|c|c|c}
\hline$n$ & $T x_{\mathrm{n}}$ & $S x_{\mathrm{n}+1}$ & $x_{\mathrm{n}+1}$ & $\left|e_{\mathrm{n}}\right|$ \\
\hline 0 & 1.7165256996 & 1.7165256996 & 0.8582628498 & 0.1417371502 \\
1 & 1.9227433021 & 1.9227433021 & 0.9613716511 & 0.1031088013 \\
2 & 1.7725085479 & 1.7725085479 & 0.8862542740 & 0.0751173771 \\
3 & 1.8819684975 & 1.8819684975 & 0.9409842488 & 0.0547299748 \\
$\vdots$ & $\vdots$ & $\vdots$ & $\vdots$ & $\vdots$ \\
69 & 1.8358097987 & 1.8358097987 & 0.9179048993 & 0.0000000001 \\
70 & 1.83580979876 & 1.83580979876 & 0.9179048993 & 0.0000000000 \\
\hline
\end{tabular}

Table 52: Jungck Mann iteration scheme $(\beta=0.98)$

\begin{tabular}{c|c|c|c|c}
\hline$n$ & $T x_{\mathrm{n}}$ & $S x_{\mathrm{n}+1}$ & $x_{\mathrm{n}+1}$ & $\left|e_{\mathrm{n}}\right|$ \\
\hline 0 & 1.7165256995 & 1.7221951856 & 0.8610975928 & 0.1389024072 \\
1 & 1.9186182640 & 1.9146898024 & 0.9573449012 & 0.0962473084 \\
2 & 1.7783624990 & 1.7810890451 & 0.8905445225 & 0.0668003787 \\
$\vdots$ & $\vdots$ & $\vdots$ & $\vdots$ & $\vdots$ \\
58 & 1.8358097986 & 1.8358097986 & 0.9179048993 & 0.0000000000 \\
\hline
\end{tabular}

Table 53: Modified Jungck Mann iteration scheme $(\beta=0.98)$

\begin{tabular}{c|c|c|c|c}
\hline$n$ & $T x_{\mathrm{n}}$ & $S x_{\mathrm{n}+1}$ & $x_{\mathrm{n}+1}$ & $\left|e_{\mathrm{n}}\right|$ \\
\hline 0 & 1.7165256995 & 1.8358443705 & 0.9179221852 & 0.0820778148 \\
1 & 1.8357845916 & 1.8358096186 & 0.9179048093 & 0.0000173760 \\
2 & 1.8358099299 & 1.8358097996 & 0.9179048998 & 0.0000000905 \\
3 & 1.8358097979 & 1.8358097986 & 0.9179048993 & 0.0000000005 \\
4 & 1.8358097986 & 1.8358097986 & 0.9179048993 & 0.0000000000 \\
\hline
\end{tabular}

Table 54: Jungck Ishikawa iteration scheme $(\alpha=0.9876$ and $\beta=0.3)$

\begin{tabular}{c|c|c|c|c}
\hline$n$ & $T x_{\mathrm{n}}$ & $S x_{\mathrm{n}+1}$ & $x_{\mathrm{n}+1}$ & $\left|e_{\mathrm{n}}\right|$ \\
\hline 0 & 1.7165256995 & 1.7809184378 & 0.8904592189 & 0.1095407811 \\
1 & 1.8758372895 & 1.8541530315 & 0.9270765157 & 0.0366172968 \\
2 & 1.8224376033 & 1.8296804230 & 0.9148402115 & 0.0122363042 \\
$\vdots$ & $\vdots$ & $\vdots$ & $\vdots$ & $\vdots$ \\
20 & 1.8358097986 & 1.8358097986 & 0.9179048993 & 0.0000000000 \\
\hline
\end{tabular}


Table 55: Modified Jungck Ishikawa iteration scheme $(\alpha=0.9876$ and $\beta=0.3)$

\begin{tabular}{c|c|c|c|c}
\hline$n$ & $T x_{\mathrm{n}}$ & $S x_{\mathrm{n}+1}$ & $x_{\mathrm{n}+1}$ & $\left|e_{\mathrm{n}}\right|$ \\
\hline 0 & 1.7165256995 & 1.8359669942 & 0.9179834971 & 0.0820165029 \\
1 & 1.8356951843 & 1.8358098261 & 0.9179049130 & 0.0000785841 \\
2 & 1.8358097786 & 1.8358097986 & 0.9179048993 & 0.0000000137 \\
3 & 1.8358097986 & 1.8358097986 & 0.9179048993 & 0.0000000000 \\
\hline
\end{tabular}

\section{Conclusions}

The modified Jungck Mann and modified Jungck Ishikawa iteration schemes for solving nonlinear equations are established. The examples discussed above show that the efficiencies of both the iteration schemes are very good as compared to the Jungck, Jungck Mann and Jungck Ishikawa iteration schemes.

\section{References}

[1] V. Berinde, On the approximation of fixed points of weak contractive mappings, Carpathian J. Math., 19 (2003), 7-22.

[2] S.K. Chatterjea, Fixed-point theorems, C. R. Acad. Bulgare Sci., 25 (1972), 727-730.

[3] S. Ishikawa, Fixed point by a new iteration method, Proc. Amer. Math. Soc., 44 (1974), 147-150, doi: 10.1090/S0002-9939-1974-0336469-5

[4] G. Jungck, Commuting mappings and fixed points, Amer. Math. Monthly, 83 (1976), 261-263, doi: 10.2307/2318216

[5] S.M. Kang, A. Rafiq, Y.C. Kwun, A new second-order iteration method for solving nonlinear equations, Abstr. Appl. Anal., 2013 (2013), Article ID 487062, 4 pages, doi: 10.1155/2013/487062

[6] R. Kannan, Some results on fixed points, Bull. Calcutta Math. Soc., 60 (1968), 71-76.

[7] W.R. Mann, Mean value methods in iteration, Proc. Amer. Math. Soc., 4 (1953), 506-510, doi: 10.1090/S0002-9939-1953-0054846-3 
[8] M.O. Olatinwo, Some stability and strong convergence results for the Jungck-Ishikawa iteration process, Creat. Math. Inform., 17 (2008), 3342 .

[9] M.O. Olatinwo, C.O. Imoru, Some convergence results for the JungckMann and the Jungck-Ishikawa iteration processes in the class of generalized Zamfirescu operators, Acta Math. Univ. Comenian., 77 (2008), 299-304.

[10] S.L. Singh, C. Bhatnagar, S.N. Mishra, Stability of Jungck-type iterative procedures, Int. J. Math. Math. Sci., 19 (2005), 3035-3043, doi: 10.1155/IJMMS.2005.3035

[11] T. Zamfirescu, Fixed point theorems in metric spaces, Arch. Math. (Basel), 23 (1972), 292-298. 